Keywords: Adolescent, safety, social determinants, health behaviours.

Health problems caused by the inadequacy of road safety measures have increased significantly and represent the main cause of morbidity and mortality among young people. A quantitative, descriptive and transversal study was performed contributing with information on safety and violence behaviours of adolescents from a school community in Vila Nova de Famalicão. A self-administered questionnaire was applied to 1,614 adolescents attending secondary and higher education in the academic year 2013/2014, aged between 14-21 years. From total participants, $56.9 \%$ were female and $86.5 \%$ attended secondary school. Results showed that $34.5 \%$ travelled by motorcycle and $65.8 \%$ by bicycle, without a safety helmet. The young drivers (3.6\%) did not always used the safety belt and $4.8 \%$ were driving after alcohol intake. Data shows a significant percentage of young people having road risk behaviours, highlighting the need for intervention in this area.

\title{
P028 - Adolescents with congenital heart disease - transition from paediatric to adult care units
}

\author{
Fernanda Carvalho (Portugal); \\ Figueiredo (Portugal) ${ }^{1}$
}

${ }^{1}$ Escola Superior de Enfermagem do Porto

Theme: Transition of care.

Keywords: Adolescents, congenital heart disease, transition.

Most children with congenital heart disease will need specialized medical care for life.

Programmes to promote transition from children's to adult care services must happen at any time and have been developed worldwide.

The purpose of this presentation is to identify the expectations of adolescents, their parents and healthcare team about the transition to adult care and to describe current practice of transition in Portugal.

A questionnaire was created, based on published evidence and international guidelines, with three versions: adolescents, parents, and healthcare professionals. It was validated by a panel of experts to evaluate the relevance and simplicity of the questions and to analyse its adequacy to the study purposes.

Questionnaires have been applied to adolescents, parents and healthcare professionals in the outpatient services of the paediatric cardiology reference centres in Portugal.

Results of this study will allow the planning of a transition program with input from the three groups involved. 